\title{
Self-Efficacy Theory as a Framework For Interventions That Support Parents of NICU Infants
}

\author{
Young Seok Lee \\ Applied Research Center \\ Motorola Mobility Inc. \\ Libertyville, IL USA \\ younglee@motorola.com
}

\author{
Craig Garfield, Hyung Nam Kim \\ Feinberg School of Medicine \\ Northwestern University \\ Chicago, IL USA \\ \{c-garfield, hyungnam.kim\}@northwestern.edu
}

\begin{abstract}
Transitioning a Very Low Birth Weight (VLBW) premature infant from the Neonatal Intensive Care Unit (NICU) to home is a very stressful task for parents. Few studies examined the needs of parents of VLBW infants during the transition; moreover, even less is known about technology development strategies that aim to increase the parenting confidence. In this study, we used Bandura's self-efficacy theory as a framework to understand ways to develop successful interventions for parents of VLBW infants. The self-efficacy theory posits that parenting behavior and the quality of care can be improved by supporting the four major sources of self-efficacy: mastery experiences, vicarious experiences, social persuasion, and physiological responses. We describe self-efficacy theory and its role in the development of technology interventions to support parents of NICU infants using a case study, called NICU-2-HOME.
\end{abstract}

Keywords-component; Neonatal Intensive Care Unit; selfefficacy theory; very low birth weight infants; design framework

\section{INTRODUCTION}

Premature births now occur in more than 12 percent of pregnancies in the United States, a 36\% increase since the early 1980s [1]. Most premature infants are born with Very Low Birth Weight (VLBW), and they are kept alive in the Neonatal Intensive Care Unit (NICU) typically for three months until they become medically stable. With the introduction of new technology in the NICU, the survival rate of VLBW infants has increased to $85 \%$, but these medically fragile infants typically live with more severe diagnoses and chronic diseases, requiring more medical attention and care. These VLBW infants (defined as those weighting less than 1500 grams at birth) have the longest average length of hospital stay as well as the highest rates of morbidity and re-hospitalization compared to all neonates [2]. The VLBW infants also have the highest use of special outpatient services, including speech and language therapies, physical and occupational therapies, neurodevelopmental evaluations, and early intervention programs.

With their baby's admission to the NICU, parents of VLBW infants undergo stressful and terrifying experiences. Most NICU admissions are unexpected and very often occur under emergent situations. Parents are therefore required to quickly adjust from the dream that they had for their baby's birth to the new reality of a NICU admission [3]. Parents are often intimidated by the unfamiliar intensive care settings that include various medical instruments, procedures, and hospital jargon. While admission to the NICU can be a difficult for parents, transitioning from the NICU to home with a high- risk infant is also stressful. Many VLBW infants are discharged with special health care needs, including home oxygen and monitoring, gastrostomy tube feeds, and several medications requiring multiple daily doses. Parents of these infants must develop and maintain a strong understanding of their infants' sensory, motor, and medical needs in order to be prepared for home caregiving. At the end of the NICU stay, parents are expected to assume full responsibility for their child in such tasks as monitoring the infant for changes in health and maintaining feeding, medication, follow-up appointments, and outpatient treatment regimens [4]. It is no surprise that parents of VLBW infants are apprehensive as they face discharge after their 2-3 months in the NICU [3]. Previous studies report parents of VLBW infants feeling ill-prepared, uninformed, and unskilled to provide a myriad of home health care tasks $[3,4]$.

To address this problem, we are conducting a research study that aims to develop technology interventions to improve parental self-efficacy in caring for their medically vulnerable infants. A few existing studies examined technology solutions to support parents of NICU infants [5,6], but they focused on a discrete part of the transition process (e.g., the hospitalization period or the post-discharge period) and lacked a theoretical foundation. Moreover, little consideration was given to strategies to increase parents' self-efficacy. In this study, we used Bandura's self-efficacy theory as a framework to develop strategies for technological interventions that increase parenting confidence. In the present paper, we describe the use of the self- efficacy theory and its role, as a framework for the NICU-2-Home project, a case study in the development of technology interventions for this vulnerable population of NICU parents transitioning home.

\section{SELF-EFFICACY THEORY}

The concept of self-efficacy lies at the center of psychologist Albert Bandura's social cognitive theory [7]. Selfefficacy is defined as "the belief in one's capabilities to organize and execute the courses of action required to manage prospective situations" (1995, p. 2). In other words, selfefficacy is a person's belief in his or her ability to succeed in a particular situation. Bandura described these beliefs as determinants of how people think, behave, and feel [7]. For

Identify applicable sponsor/s here. (sponsors) 
instance, individuals with high self-efficacy tend to view challenging problems as tasks to be mastered, develop deeper interest in solving the problem, and maintain a greater effort to successfully complete the task. Conversely, individuals with low self- efficacy are likely to focus on personal failure and negative outcomes. They easily lose confidence in their capabilities and try to avoid challenging tasks as a result. According to Bandura [7], self-efficacy values are influenced by four resources: mastery experiences, vicarious experiences, social persuasion and physiological states (Figure 1).

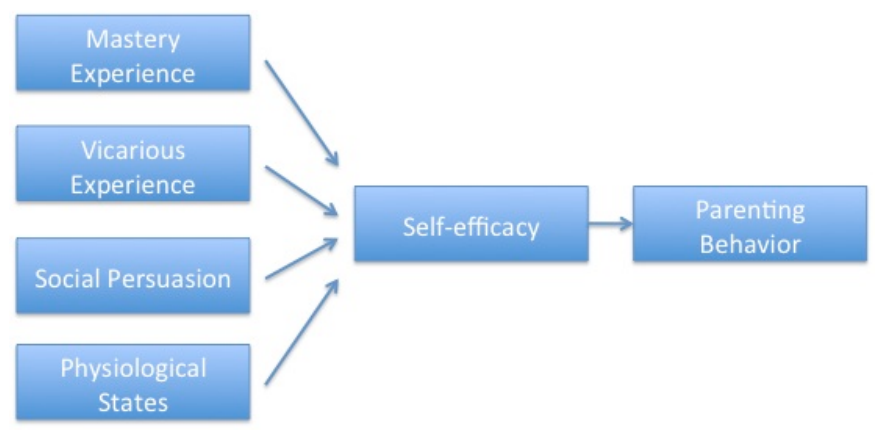

Figure 1. Bandura's Self-Efficacy Theory Applied to The NICU-2-HOME Project

The most effective way of developing a strong sense of efficacy is through mastery experiences. Successes build a robust belief in one's personal efficacy. These successful experiences form expectations that they will succeed in other situations that may be similar or substantially different from the original experience [7]. This also implies that helping people master specific skills creates successful experiences and builds the confidence to succeed. Vicarious experiences also have an effect on self-efficacy. Bandura suggested that observing other people successfully completing a task can enhance personal self-efficacy by demonstrating that the task is "doable" with some effort [8]. According to Bandura, "Seeing people similar to oneself succeed by sustained effort raises observers' beliefs that they too possess the capabilities to master comparable activities to succeed" [7]. The third resource of self-efficacy is social persuasion. Bandura asserted that people could be persuaded to believe that they have the skills and capabilities to succeed through the use of suggestion, exhortation, or selfinstruction. Verbal encouragement from others helps people overcome self-doubt. It helps people focus on giving their best effort to the task at hand instead of being concerned with potential failure [8]. Lastly, self-efficacy can be enhanced by controlling physiological states. Moods, emotional states, physical reactions, and stress levels can all impact how a person feels about their personal abilities in a particular situation. By learning how to minimize stress and elevate mood when facing difficult or challenging tasks, people can improve their sense of self-efficacy [7,8].

Self-efficacy theory has been applied to various domains including health-related behavior, sports, and education. In the domain of healthcare, numerous studies have proven that health-related behaviors, such as non- smoking, physical exercise, and dieting, are dependent on one's perceived selfefficacy [9].

\section{CASE STUdY: NICU-2-HOME}

NICU-2-HOME is an NIH-funded research project that aims to develop a technology intervention for NICU parents. In this ongoing research, we have conducted a number of qualitative studies to gain an in-depth understanding of the current discharge practices, transition behaviors, and challenges that parents of NICU infants are faced with when they prepare to take their infant home. Our studies include rapid contextual inquiries as well as a triangulation of stakeholder data collected via phone interviews with 25 NICU parents, a focus group with six neonatologists and a focus group with five community pediatricians, followed by a literature review. During the rapid contextual inquiry, a multi-disciplinary team, consisting of a pediatric research scientist, a HCI professional, an interaction designer, and a software engineer, conducted multiple sitevisits to the NICU in an academic-community hospital system in the Chicago area. At each visit, the research team observed the work practices and information flow at the NICU. The team also interviewed the NICU staff (i.e., medical director, four nurses, two neonatologists, and one care coordinator) to understand their discharge process and experiences with parents. We also conducted phone interviews with 25 parents (15 mothers, 10 fathers) of 16 VLBW babies who had an average gestational age of 29.5 weeks and a NICU stay of $>2$ months. Parents' average age was 31 years; $68 \%$ were White, $20 \%$ Hispanic and 12\% Black. These interviews focused on their descriptions and experiences with the discharge and transition process, including communication and information exchange with the NICU staff and pediatricians. We also conducted two separate focus groups with six neonatologists and six pediatricians.

\section{THEORY-DRIVEN DESIGN IMPLICATIONS}

Bandura's theory suggests that stronger parenting selfefficacy would result in an improved ability for parents to care for their VLBW NICU infants, and supporting four unique resources can develop a strong sense of parental self-efficacy. In the following we describe NICU parents' inherent needs and summarize design implications for technological interventions suggested by self-efficacy theory.

\section{A. Implications for Mastery Experiences}

According to Bandura [7], helping parents master specific skills and knowledge in caregiving tasks creates successful experiences and builds the confidence to succeed. VLBW NICU infants spend 2-3 months in the NICU, with most of that time being in a temperature-controlled isolette. Around the $37^{\text {th }}$ week gestation (adjusted), most infants are mature enough to transition into an open crib without assisted regulation of their temperature. It is at this point that discharge training begins in earnest as most infants will be discharged in about 2 weeks.

In this discharge preparation process, parents of VLBW infants receive intensive education on care-giving tasks, such as identification of post discharge needs, specialized care for specific disorders of prematurity, coordination of outpatient 
supplies and medications, referrals to appropriate services, transferring the medical care to the pediatric provider, and daily care practices (e.g., changing diaper, feeding, medication preparation). The NICU staff responsible for discharge education, mainly nurses and social workers, lead the education and encourage parents' active participation and hands-on practice instead of simply "showing" or "telling". Despite these efforts, parents' needs for information and communication are often overlooked during the discharge preparations. Thus, parents often feel unprepared for discharge and desire more information and greater communication than is provided to them.

For example, parents of VLBW infants receive a great deal of information upon admission to the NICU. A 150-page booklet full of information on general premature infant care is given to each parent and it is assumed that they are likely to have read it by the time of discharge. The parents are given even a larger amount of additional information during the twoweek discharge preparations, ranging from post discharge home care instructions to coordination of outpatient supplies and services. However, we found that such an expanse of information may not always meet parents' individual needs, nor is it easily retrievable or accessible. Currently, educational materials are rarely tailored to a specific baby's condition or parents' health literacy. Parents, therefore, find the educational material "very general" or "irrelevant" or "difficult to understand". The traditional paper-based information packet also makes dynamic information delivery and immediate information search difficult. As a result, parents are seeking more interactive form of information. As one father remarked in the interview: "What's in this booklet should be presented in a more interactive form. That would be nice, yeah. You could make the whole audio and visual product out of this book. Especially what would be helpful is the physical therapy. Using a video it would be much easier to learn this.. like, 'Ok, this is sign A, ok, this is how your baby looks.' A picture is one thing, but if you had a little video of each of these positions they want you to put him in, or the signs you should be looking at, yeah, that would be cool."

In another example, in the NICU studied in this research, the discharge preparations are classified into a 34 items of todo-list and this "discharge checklist" is one means to document parents' mastery of the necessary skills and information acquisition needed prior to discharge (Fig. 2). However, the checklist is printed on a tiny yellow card $(90 \times 75 \mathrm{~mm})$ enlisting the title of the 34 items without detailed descriptions of medical acronyms (e.g., PKU, ABR/BSAER). Taped at the cribside and checked off by nurses, this discharge checklist was designed to be both a nurse's aid and a visual map to check parents' discharge preparation progress, but we found that parents themselves did not understand the overall flow of the discharge process nor the medical terms written on the list.

Self-efficacy theory suggests it is important to help parents master specific skills and knowledge in caregiving tasks to build the confidence to succeed. Despite NICU parent's enthusiam about learning, we found there lacks a sufficient tool to facilitate their education. It is our belief that pervasive technologies including mobile technologies can be an important asset to encourage and support parents' self-learning while accommodating individual differences such as unique learning styles and health literacy level, and culture differences among ethnic groups. For instance, an interactive discharge checklist can be developed for cribside tablets or in-room kiosks to provide parents with anticipatory guidance on learning or their baby's conditions and medical treatment. Using this system, parents will be able to access educational material repeatedly and at their convenience.

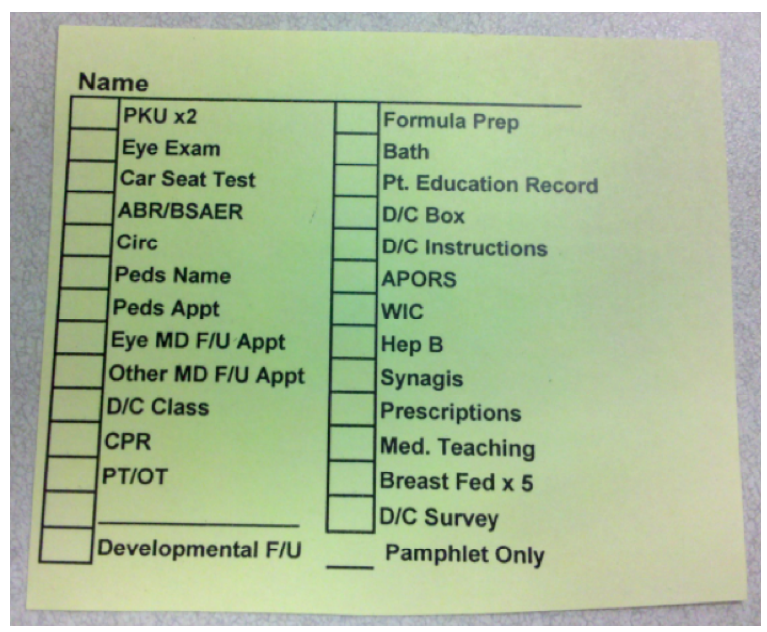

Figure 2. Discharge Checklist

With respect to the lack of personalized education material, technology could also contribute to creating effective education materials that are tailored to a specific baby's condition, parents' demographic variables, personality profile, or health literacy. Recently, researchers in Natural Language Generation (NLG) have begun to apply methods from Artificial Intelligence and Computational Linguistics to develop automated systems for tailoring health information to individual patients [10]. Parallel work has explored automatic generation of short micro-explanations that summarize medical events for real-time delivery in clinical settings [11]. This type of application in the NICU would be of immense benefit for parents of VLBW infants.

In addition, interactive multimedia strategies delivered through video or electronic channels may be another approach for effective parent education. A repository of media-based education material can be built in the form of a web service that is accessible to parents any time - even in the post-discharge period - using various computing devices (e.g., smart phones, in-room kiosks, tablets, and PCs). The repository could contain educational materials such as discharge class materials, CPR instructions, car seat installation instructions, feeding, bathing, proper preparation of formula/breast milk, symptom evaluation and management, mediation information, and resources in the community.

\section{B. Vicarious Experiences and Social Persuasion}

Vicariously observing other parents successfully completing a task or receiving the verbal encouragement from others is another way to positively affect self-efficacy. NICU parents seem especially interested in both real and digital vicarious experiences. They were highly interested in the 
"NICU parent support group" that would connect them to other parents whose infants already graduated from the NICU. Using such support groups, parents expected to receive informational and emotional support from the parents who had "been there, done that". Many NICU parents utilized a range of blog websites, such as caringbridge.org and carepages.com for vicarious experiences while exchanging information and social persuasion with parents in a similar condition. However, parents desired blog sites that are specifically designed for NICU infants and families rather than general websites with mixture information of full-term infants. Also, they desired a connection with other parents who are dealing with the same medical and environmental condition. For example, parents of multiples wish to be connected with other parents of multiples, or parents who lost one of their infants wish to get emotional support from families in the same case.

The above findings suggest that online communities using social media technology would be a great benefit for NICU parents in finding other parents in similar conditions and sharing verbal encouragement. Such social outlets should also provide an easy way for the parents to add infant's health updates, diagnoses, and photos to facilitate interaction between the members of a NICU parent support group. Recording and sharing their experiences with others in a timely and efficient manner can be challenging; therefore, mobile phone applications, such as a mobile multimedia care diary that allows parents to keep a record of nursing/care (e.g., feeding length, supplementation, bowel movements, wet diaper frequency) as well as infants' developmental data (e.g., weight and height), could help smooth data capturing and sharing. Tools that support lightweight communication among the social support network, such as text/multimedia message, could make a considerable contribution to increase the frequency and reach of the social persuasion as well.

\section{Physiological Responses}

Parenting a VLBW infant and transitioning this child is stressful work. Parents must balance other household responsibilities along with caring for the NICU graduate, all of which can be a source of anxiety and feature for parents. In our case study, several parents reported high stress level and practice of a deep breathing technique when stressed at home. Self-efficacy theory asserted that physiological or emotional states such as stress, anxiety, or an individual's mood also affect judgments about parental self-efficacy [7]. Therefore, it is important to promote positive emotional states of parents, and pervasive technologies provide various opportunities for this role. For example, mobile applications can be developed to assess parents' stress level in their daily lives and provide remedies to alleviate the stress. Validated stress assessment tools, such as the Parental Stressor Scale-Neonatal Intensive Care [12], can be implemented with a smartphone, allowing parents to assess their stress level by themselves and receive proper consultations from an application that teaches deep breathing, relaxation, and meditation technique. Alternatively, wireless sensors including Galvanic Skin Response provide an opportunity for measuring the stress level as a valid biological marker [13]. Lastly, mobile applications that help share the stress level between spouses would be beneficial to promote the positive family coping and relationship.

\section{CONCLUSION}

We described self-efficacy theory and its role in the development of high-level design strategies for interventions that aims to increase NICU parents' self-efficacy. Badura's theory provides a theoretical foundation to improve parental self-efficacy in caring for their medically vulnerable infants as well as a systematic guidance to design technologies to support the four main sources of parental self-efficacy. We shared the broad contexts of the discharge process and challenges faced by NICU parents as surfaced during our case study. These findings lay a foundation for designing technologies that empower and provide informational, emotional, and social support to this vulnerable population.

\section{REFERENCES}

[1] B. E. Hamilton, J. Martin, and S J. Ventura, Births: Preliminary Data for 2008. National Center for Health Statistics. Hyattsville, MD. 2010.

[2] M. A. Underwood, B. Danielsen, W. M. Gilbert, "Cost, causes and rates of rehospitalization of preterm infants", J. Perinatol. 27(10), pp.614-619. 2007

[3] K. Dyer, NICU parent support blog. Retrieved June 28, 2011, from http://nicuparentsupport.blogspot.com/2007_07_01_archive.html

[4] N. Sneath, "Discharge Teaching in the NICU: Are Parents Prepared? An Integrative Review of Parent's Perceptions. Neonatal Network." J. Neonatal Nursing. 28 (4), pp. 237-246. 2009.

[5] J. Gray, C. Safran, R. Davis, et al. "Baby CareLink: Using the Internet and Telemedicine to Improve Care for High-Risk Infants". Pediatrics. 106(6), pp.318-1324, 2000.

[6] G. R. Hayes, K. Cheng, S. Hirano, S. Park, D. Gravem, J. Rich, and D. Cooper, "FitBaby: Using observations of daily living to improve the health of preterm infants and their caregivers." In Proc. workshop on Interactive Systems in Healthcare, pp.73-76.2010.

[7] A. Bandura, Self-Efficacy in Changing Societies. Cambridge University Press. 1995.

[8] A. Bandura, "Self-efficacy". Ed. V. S. Ramachaudran, Encyclopedia of human behavior, 4. New York: Academic Press, 1994, pp. 71-81.

[9] M. Conner and P. Norman, "Predicting health behaviour: A social cognition approach". In Predicting Health Behaviour: Research and Practice with Social Cognition Models, M. Conner and P. Norman, Ed. 2nd edition. Buckingham: Open University Press. 2005.

[10] Di Marco, et al. "Authoring and generation of individualised patient education materials”. In Proc. AMIA SYMP 2006, pp. 195-199.

[11] L. Wilcox, D. Morris, D. Tan, J. Gatewood, and E. Horvitz, "Characterizing Patient-Friendly "MicroExplanations" of Medical Events.” In Proc. CHI 2011, ACM Press, 2011, pp. 29-32.

[12] M. S. Miles, S. G. Funk, S. G., and J. Carlson, J. "Parental stressor scale: Neonatal intensive care unit” Nursing Research. 42(3), 148-152, 1993.

[13] T. J. Dishongh and M. McGrath, Wireless Sensor Network. Artechn House.Boxton. 2010. 